\title{
Internet based vascular risk factor management for patients with clinically manifest vascular disease: randomised controlled trial
}

\author{
() (1) $\Theta$ OPEN ACCESS
}

\author{
J W P Vernooij PhD student, internist in training ${ }^{1}, \mathrm{H}$ A H Kaasjager internist ${ }^{2}, \mathrm{Y}$ van der Graaf \\ professor $^{3}$, J Wierdsma nurse practitioner ${ }^{1}, \mathrm{H} \mathrm{M} \mathrm{H}$ Grandjean nurse practitioner ${ }^{2}$, M M C Hovens \\ internist $^{2}, \mathrm{G}$ A de Wit associate professor ${ }^{3}, \mathrm{~F} \mathrm{~L} \mathrm{~J} \mathrm{Visseren} \mathrm{professor}{ }^{1}$, on behalf of the SMART \\ study group
}

${ }^{1}$ Department of Vascular Medicine, University Medical Center Utrecht, PO Box 85500, 3508 GA Utrecht, Netherlands; ${ }^{2}$ Department of Internal Medicine, Rijnstate Hospital, Arnhem, Netherlands; ${ }^{3}$ Julius Center for Health Sciences and Primary Care, University Medical Center Utrecht

\begin{abstract}
Objective To investigate whether an internet based, nurse led vascular risk factor management programme promoting self management on top of usual care is more effective than usual care alone in reducing vascular risk factors in patients with clinically manifest vascular disease.

Design Prospective randomised controlled trial.

Setting Multicentre trial in secondary and tertiary healthcare setting.

Participants 330 patients with a recent clinical manifestation of atherosclerosis in the coronary, cerebral, or peripheral arteries and with at least two treatable risk factors not at goal.

Intervention Personalised website with an overview and actual status of patients' risk factors and mail communication via the website with a nurse practitioner for 12 months; the intervention combined self management support, monitoring of disease control, and drug treatment.

Main outcome measures The primary endpoint was the relative change in Framingham heart risk score after 1 year. Secondary endpoints were absolute changes in the levels of risk factors and the differences between groups in the change in proportion of patients reaching treatment goals for each risk factor.

Results Participants' mean age was 59.9 (SD 8.4) years, and most patients ( $n=246 ; 75 \%)$ were male. After 1 year, the relative change in Framingham heart risk score of the intervention group compared with the usual care group was $-14 \%$ ( $95 \%$ confidence interval $-25 \%$ to $-2 \%$ ). At baseline, the Framingham heart risk score was higher in the intervention group than in the usual care group (16.1 (SD 10.6) $v 14.0$ (10.5)), so the outcome was adjusted for the separate variables of the Framingham heart risk score and for the baseline Framingham heart
\end{abstract}

risk score. This produced a relative change of $-12 \%(-22 \%$ to $-3 \%)$ in Framingham heart risk score for the intervention group compared with the usual care group adjusted for the separate variables of the score and $-8 \%(-18 \%$ to $2 \%)$ adjusted for the baseline score. Of the individual risk factors, a difference between groups was observed in low density lipoprotein cholesterol $(-0.3,-0.5$ to $-0.1, \mathrm{mmol} / \mathrm{L})$ and smoking $(-7.7 \%$, $-14.9 \%$ to $-0.4 \%$ ). Some other risk factors tended to improve (body mass index, triglycerides, systolic blood pressure, renal function) or tended to worsen (glucose concentration, albuminuria).

Conclusion An internet based, nurse led treatment programme on top of usual care for vascular risk factors had a small effect on lowering vascular risk and on lowering of some vascular risk factors in patients with vascular disease.

Trial registration Clinical trials NCT00785031.

\section{Introduction}

Patients with a recent clinical manifestation of a vascular disease (such as myocardial infarction, stroke, or peripheral arterial disease) are at increased risk for developing a new vascular event or death. ${ }^{1}$ More patients survive an acute vascular event nowadays, and as a consequence the total number of patients in the chronic phase of vascular disease is increasing. Established strategies to reduce vascular risk are treating hypertension, reducing low density lipoprotein cholesterol, using platelet inhibitors, controlling weight, stopping smoking, and increasing physical exercise..$^{2-5}$ Treatment of these risk factors, alone or in combination, has been shown to be very effective in reducing the risk of recurrent vascular events (myocardial 
infarction, ischaemic stroke) and death. ${ }^{6}$ However, in daily clinical practice, treatment goals are often not reached. In a prospective cohort study in patients with established vascular disease or type 2 diabetes, 1.5 years after referral to the hospital and even after participation in a risk factor screening programme, prevalences were $43 \%$ for hypertension, $40 \%$ for hypercholesterolaemia, $24 \%$ for obesity, and $19 \%$ for smoking. ${ }^{7}$ Comparable numbers are seen in patients with coronary artery disease, ${ }^{8}$ indicating that a large proportion of patients with a clinical manifestation of a vascular disease are still at high residual cardiovascular risk as a result of not reaching treatment targets as advocated in (inter)national guidelines.

Treatment of vascular risk factors by nurse practitioners has been shown to be effective in reducing cardiovascular risk factors and vascular risk, ${ }^{9}{ }^{10}$ but this treatment is costly and time consuming for patients and healthcare professionals, as frequent visits to the outpatient clinic are needed. Stimulating self management has been shown to be effective in lowering blood pressure, ${ }^{11}$ and supporting self management with an internet programme may add to the effectiveness of the nurse practitioner's intervention. Use of the internet is a low cost method compared with a regular outpatient clinic. Experience and evidence exist for effective internet based treatment of depression, ${ }^{12}{ }^{13}$ physical activity in patients with rheumatoid arthritis, ${ }^{14}$ pain reduction in patients with fibromyalgia, ${ }^{15}$ reduced mortality and hospital admission in patients with heart failure, ${ }^{16}$ and glucose control in patients with type 2 diabetes. ${ }^{17-19}$ In a small, single centre, uncontrolled, pilot study, an internet based and nurse led vascular risk reduction programme on top of usual care was feasible and showed beneficial effects on risk factor levels after six months. ${ }^{20}$ The objective of the multicentre, randomised trial reported here was to evaluate the effect at one year of an internet based, nurse led programme for management of vascular risk factors on vascular risk and vascular risk factors in patients with clinically manifest vascular disease.

\section{Methods \\ Trial design}

The study was a multicentre, prospective, randomised controlled trial comparing intervention via the internet plus usual care with usual care alone. The local study coordinator used an online randomisation procedure with a printed confirmation. The overall study coordinator checked participants' assignment with the printed confirmation.

\section{Study population}

All randomised patients had a recent clinical manifestation of atherosclerosis in the coronary, cerebral, or peripheral arteries diagnosed and were referred by their vascular specialist (vascular surgeon, cardiologist, neurologist) or their general practitioner at the Rijnstate Hospital Arnhem, the Netherlands, a teaching hospital, and at the University Medical Center Utrecht, Utrecht, the Netherlands.

Vascular risk factors were measured as part of routine clinical practice. Patients aged between 18 and 80 years were eligible for participation in the study if at least two of the following six treatable risk factors were not at target: systolic blood pressure above $140 \mathrm{~mm} \mathrm{Hg}$, low density lipoprotein cholesterol above $2.5 \mathrm{mmol} / \mathrm{L}$, triglycerides above $1.7 \mathrm{mmol} / \mathrm{L}$, body mass index above 25 , diabetes or fasting glucose above $6.1 \mathrm{mmol} / \mathrm{L}$, and smoking. Patients had to be able to read and write Dutch, to be independent in daily activities (Rankin score $<3$ ), ${ }^{21} 22$ and to have access to the internet at home. Patients with an estimated life expectancy less than two years or a malignant disease were not included in the study.

\section{Internet based, nurse led risk factor programme and usual care}

Patients who met the inclusion criteria and did not met the exclusion criteria were invited to participate in the study. After giving written informed consent, patients were randomised to receive either internet based care or usual care.

Patients randomised to the internet programme received an internet based programme of vascular risk factor management on top of usual care during one year. For this purpose, a website was constructed and tested in a pilot study, ${ }^{20}$ and several improvements were made on the basis of evaluation of the website by patients in the pilot study. The website was developed using Microsoft Visual Studio 2005 (development environment) in Visual C\# (programming language). It uses a Microsoft SQL Server 2005 database for data storage. The scripts for the website can be obtained from the corresponding author. At the start, patients were invited for a one hour visit to the outpatient clinic of the nurse practitioner in the hospital. At this visit, patients received information on their risk factor levels, instructions about the internet programme, and a username and password for their personalised website. Subsequent contacts between patient and nurse practitioner were through the internet, and no further outpatient clinic visits were scheduled. Depending on the presence of risk factors that needed (additional) treatment, the nurse practitioner personalised the website for each individual patient, taking on average 10 minutes. The opening page showed an overview of the actual status of all risk factors (green=at goal, yellow=close to goal, red=needs attention) and drug use (supplementary figure A). Within the personalised website, each risk factor was displayed on a separate internet page containing a history of risk factor measurements (such as blood pressure or low density lipoprotein cholesterol), drug use, treatment goal, advice from the nurse, correspondence between nurse and patient, and news items for that particular risk factor (supplementary figure B). Patients were instructed to use the website as frequently as considered convenient and necessary at their own discretion and to log in at least every other week to submit new measurements (blood pressure, weight, smoking status, cholesterol) and to read and send messages.

The internet programme was linked to the website of the University Medical Center Utrecht for general information on risk factors and vascular diseases. The nurse practitioner was able to view all files and pages from all patients and had access to pages with a total overview of the current status of risk factors, last log-in attempts of each patient, and new messages sent by patients. The treating nurse practitioner logged in every working day and replied to messages sent by patients and sent messages to patients not using the programme at least every other week. In case of non-response by a patient, the nurse contacted patients by phone. Patients were encouraged to measure their own blood pressure at home or ask the general practitioner to measure their blood pressure. Patients were free to use their own device, as this reflects daily clinical practice. For measuring plasma lipids and glucose, patients received laboratory forms from the nurse by mail for blood to be drawn in their own city or at the University Medical Center Utrecht or Rijnstate Hospital Arnhem, whichever was convenient for the patient. The nurse practitioner worked according to the 2006 Dutch cardiovascular risk management guideline, ${ }^{23}$ which is closely related to the 2003 ESC/EAS guidelines ${ }^{24}$ for the diagnosis and treatment of vascular risk factors. Internists supervised the nurse practitioners. Changes in drug regimen 
were made by sending prescriptions to patients by regular mail. The care delivered by the nurse practitioner with the internet programme was on top of usual care and did not replace the care given by the treating physician in the hospital and the general practitioner.

The usual care given by the medical specialist or general practitioner was also based on the 2006 Dutch cardiovascular risk management guideline. The guideline was applicable for primary care and hospital care. Patients randomised to usual care were asked to contact their treating physician (vascular surgeon, cardiologist, neurologist) at the hospital or the general practitioner for risk factor management. The treating physician and the general practitioner were also informed of the risk factor status in writing. The treating physician was free to determine the frequency of control. This could range from an annual visit for a patient with stable coronary artery disease to every three months for a patient with type 2 diabetes mellitus.

\section{Study measurements}

At baseline, drug use, exercise, and smoking were registered; blood pressure, weight, height, and waist circumference were measured; fasting blood was drawn for measurement of lipids, glucose, and creatinine; and urine was analysed for albuminuria. After 12 months, all patients in both groups were asked to return to the clinic for the same measurements. Independent research nurses not aware of the treatment allocation of the patients made the follow-up measurements. We used the four variable modification of diet in renal disease (MDRD) equation to calculate estimated glomerular filtration rate. ${ }^{25} \mathrm{We}$ defined albuminuria as a urine albumin:creatinine ratio of $2.5 \mathrm{mg} / \mathrm{mmol}$ or above in men and $3.5 \mathrm{mg} / \mathrm{mmol}$ or above in women. ${ }^{26} \mathrm{We}$ defined type 2 diabetes mellitus as a referral with a diagnosis of type 2 diabetes mellitus, use of glucose lowering drugs, or a fasting glucose concentration of at least $7.0 \mathrm{mmol} / \mathrm{L}$ at screening.

\section{Primary and secondary endpoints}

The primary endpoint was the relative change in Framingham heart risk score after one year. ${ }^{27}$ This was calculated as the difference between the groups in change in Framingham heart risk score from baseline to one year follow-up: ((baseline Framingham heart risk minus follow-up Framingham heart risk in usual care group) minus (baseline Framingham heart risk minus follow-up Framingham heart risk in intervention group)) divided by the mean Framingham heart risk score at baseline. We calculated the Framingham heart risk score for each individual patient at baseline and after one year's follow-up on the basis of actual risk factor levels. The Framingham heart risk score represents the predicted 10 year risk for coronary heart disease and was developed for patients free of vascular disease. In this study, we used the Framingham heart risk score as a summary score of vascular risk factors. ${ }^{28}$ The estimated absolute risk level by Framingham heart risk cannot be regarded as a precise reflection of actual risk, ${ }^{29}$ but absolute change in Framingham heart risk over time is likely to reflect absolute changes in risk.

The secondary endpoints were the differences between the intervention and usual care groups in the absolute changes in the levels of risk factors (level at one year minus level at baseline) and the differences between groups in the change in the proportion of patients reaching treatment goals for each risk factor.

\section{Sample size calculation}

For the primary outcome, we aimed to detect a $10 \%$ relative difference in the Framingham heart risk score between the two groups on the basis of a pilot study. ${ }^{20} \mathrm{We}$ considered this to be a minimal clinically relevant difference. In the pilot study $(\mathrm{n}=50)$, we found a change in Framingham heart risk score from 11.2 (SD 7.8) to 9.0 (5.8): difference -2.2 (6.0). For the study reported here, we recruited patients from among participants in an ongoing cohort study with a higher mean age than the patients in the pilot study and a higher Framingham heart risk score of $20 \%$. For the sample size calculation, we used an absolute change in Framingham heart risk score of -2.0 (6.0), an $\alpha$ of 0.05 , and a power of $80 \%$. The calculated sample size was 146 patients in each group.

\section{Adverse events and clinical endpoints}

During follow-up, patients were asked to complete a questionnaire by internet every three months, to report newly diagnosed diseases and hospital admissions. When a cardiovascular event was suspected, we retrieved patients' medical records and documentation from their treating specialist or general practitioner. Three independent non-treating specialists separately assessed suspected vascular events and mortality. Registered events included vascular interventions (such as percutaneous coronary artery interventions), stroke, myocardial infarction, vascular mortality, and other severe adverse events (all events causing death, life threatening events, events requiring at least one night of hospital stay or prolonged hospital stay, or events causing significant invalidity or labour incapacity) and non-severe adverse events (any reported event).

\section{Data analyses}

We expressed results as means with standard deviations or as absolute numbers and percentages. We expressed non-normally distributed variables as median and interquartile range. We present absolute changes in Framingham heart risk score and individual risk factors between baseline and follow-up, with $95 \%$ confidence intervals, in complete case analyses. We also calculated the change in percentages of patients who achieved treatment goals for individual risk factors in both groups. We used an independent sample $t$ test to assess differences between groups in absolute changes in Framingham heart risk score and risk factors between baseline and 12 months' follow-up and differences between groups in the proportion of patients achieving treatment goals. We did sensitivity analyses by imputing the missing values with the last observation carried forward, truncation of extreme values, and calculation of the intervention result by linear regression with adjustment for baseline risk. We used SPSS statistics 18.0.2 for all statistical analyses.

\section{Results \\ Baseline characteristics}

Patients were recruited between October 2008 and March 2010. A total of 638 patients who fulfilled the inclusion criteria were invited for participation, of whom 330 were randomised (fig $1 \Downarrow$ ). The mean age was 59.9 (SD 8.4) years, and most patients (75\%) were male (table $1 \Downarrow$ ). All patients had a recent manifestation of vascular disease diagnosed; most often (49\%) this was coronary artery disease. Mean low density lipoprotein cholesterol was $2.8(0.9) \mathrm{mmol} / \mathrm{L}$, and mean systolic blood pressure was 140 (18) $\mathrm{mm} \mathrm{Hg}$. 
The main reason for not participating in the study was the absence of internet access or basic computer skills (58\%). Patients not randomised were slightly older $(62.1(10.7) v 59.9$ (8.4) years) and more often female $(33 \% v 25 \%)$ than randomised patients (supplementary table A).

During the study, $16(5 \%)$ patients dropped out and did not have a follow-up measurement. Those patients seemed to be of similar age to the patients with complete follow-up (59.6 (8.0) $v 59.9$ (8.4) years), were more often female (37\% v 25\%), and seemed to have a worse risk factor profile (Framingham heart risk score $18.1(19.1) v 15.0(10.6))$ (table $2 \Downarrow)$.

\section{Difference in change in Framingham heart risk score between intervention and control groups}

After one year, we found a relative change of $-14 \%(95 \%$ confidence interval $-25 \%$ to $-2 \%$ ) in Framingham heart risk score in the intervention group compared with the usual care group. At baseline, the Framingham heart risk score was higher in the intervention group than in the usual care group (16.1 $(10.6) v 14.0(10.5))$. Therefore, we used linear regression analysis to adjust the outcome for the separate variables of the Framingham heart risk score (age, sex, systolic blood pressure, low density lipoprotein cholesterol, high density lipoprotein cholesterol, type 2 diabetes mellitus, and current smoking) and for the baseline level of the Framingham heart risk score. This produced a relative change of $-12 \%(-22 \%$ to $-3 \%)$ in

Framingham heart risk score in the intervention group compared with the usual care group when adjusted for the separate variables of the Framingham heart risk score and $-8 \%(-18 \%$ to $2 \%$ ) when adjusted for the baseline level of the Framingham heart risk score (table $3 \Downarrow$ ). The range of the Framingham heart risk score was 0.7 to 60.0 .

The difference in change in low density lipoprotein cholesterol was -0.3 (95\% confidence interval -0.5 to -0.1 ) $\mathrm{mmol} / \mathrm{L}$ $(\mathrm{P}<0.001)$. This translated to a difference between groups in patients reaching the low density lipoprotein cholesterol goal of less than $2.5 \mathrm{mmol} / \mathrm{L}$ of $18.4 \%$ (5.9\% to $30.9 \%$; $\mathrm{P}=0.004$ ). In the intervention group, 8/42 (19\%) patients stopped smoking, compared with four $(10 \%)$ patients who started smoking in the usual care group, a difference between groups in change in patients who quit smoking of $7.7 \%$ (0.4 to 14.9; $\mathrm{P}=0.038$ ) (table $4 \Downarrow$ ). Some other risk factors tended to improve (body mass index, triglycerides, systolic blood pressure, renal function) or tended to worsen (glucose concentration, albuminuria).

Truncation of extreme values of the Framingham heart risk score ( $<1 \%$ and $>99 \%$ centiles) or imputation of missing values with the last observation carried forward showed similar differences in change of Framingham heart risk score between groups (table $5 \Downarrow$ ). Stratification for sex, age, type 2 diabetes mellitus, body mass index, or smoking status produced similar results (table $5 \Downarrow$ ).

\section{Use of the website by patients}

Of the 155 patients in the intervention group, 152 patients actually logged in. They logged in a median of 56 (interquartile range 35-83) times during the year (supplementary table B). Patients $(\mathrm{n}=134)$ sent a median $14(7-22)$ messages, and 131 patients entered a median 7 (3-14) measurements during the year. Measurements most often entered related to blood pressure (111 patients, median 2 (2-6)) and weight (114 patients, median 3 (1-6)). The monthly number of logins decreased during the intervention period, from a maximum in the third month with 1099 logins to 435 logins in the 12th month (supplementary figure C). Patients in the highest third of website use had the highest Framingham heart risk score (17.6 (10.7) compared 14.1 (9.1) in the lowest third). Patients in the highest third of website use had the largest change in Framingham heart risk score during the intervention $(-3.8(-6.3$ to -1.3$)$ compared with $-1.4(-3.6$ to 0.8 ) in the lowest third) (supplementary table C). During the one year intervention period, the time spent by nurse practitioners was on average 23 (12) minutes/month/patient.

\section{Vascular events and other severe adverse events}

Forty patients reported a total of 50 vascular events (table $6 \Downarrow$ ). The intervention was safe, as the hazard ratio for a subsequent vascular event was 0.66 (95\% confidence interval 0.35 to 1.24 ). The hazard ratio was 0.64 (0.34 to 1.21) after adjustment for baseline Framingham heart risk score. Vascular events occurred equally throughout the study period, as shown in the Kaplan-Meier curve (fig $2 \Downarrow$ ). Thirty-eight patients reported 47 other severe adverse events, of which five were severe bleeding events.

\section{Discussion}

This study showed that an internet based, nurse led vascular prevention programme on top of usual care compared with usual care alone resulted in a small relative reduction in the Framingham heart risk score of $14 \%$ after 12 months in patients with clinically manifest vascular disease. At baseline, the Framingham heart risk score was higher in the intervention group than in the usual care group. Therefore, we adjusted the primary outcome for the separate variables of the Framingham heart risk score and for the baseline Framingham heart risk score. The difference in the primary outcome was not statistically significant after adjustment for baseline Framingham heart risk score. The clinical importance of this effect is small and limited. A larger reduction in low density lipoprotein cholesterol occurred and a larger proportion of patients stopped smoking in the intervention group compared with the usual care group.

\section{Comparison with other studies}

Randomised controlled trials on lowering overall vascular risk with the use of internet interventions are scarce. In primary prevention, a two year randomised controlled trial using a website with education modules and personal tailored counselling support in 276 healthy overweight participants showed no significant effect on vascular risk factors, although several risk factors tended to improve. ${ }^{30}$ A cluster randomised controlled trial in 163 patients with type 2 diabetes who received the combined intervention of behavioural mobile phone and internet coaching with presentation of blood glucose concentrations, lifestyle measurements, and self management support was effective in reducing glycated haemoglobin levels after one year by $1.2 \%$ (95\% confidence interval $0.6 \%$ to $1.8 \%$ ) compared with a control group. ${ }^{17}$ In that study, no differences in blood pressure or plasma lipid concentrations were seen between groups. In a small randomised controlled study $(n=15)$ in patients after a myocardial infarction, a virtual cardiac rehabilitation programme-consisting of online intake forms; one-on-one chat sessions with a nurse, dietitian, and exercise specialist; downloadable exercise heart rate monitoring; education; and data monitoring of blood pressure, weight, and glucose-resulted in significant changes in high density lipoprotein cholesterol, triglycerides, exercise capacity, and weekly physical activity after 12 weeks. ${ }^{31}$ Evaluation of a web 
based cardiac rehabilitation programme for patients with vascular disease, consisting of email contact with a case manager, education modules assigned by the case manager, and entering of data (for example, number of minutes of exercise, blood pressure measurements), in combination with the option of participating in an online discussion group, resulted in a decrease in body mass index of 0.7 compared with a control group after six months. ${ }^{32}$ In that study, blood pressure, lipid values, and physical exercise improved as well, but this was not statistically significant.

Our multicentre randomised controlled trial is the largest study in this field to date. Sensitivity analysis showed a comparable result in subgroups, and the intervention could probably be widely implemented. The results of this study can be generalised to patients with access to the internet at home and with sufficient computer skills. The number of patients without internet access or with no computer skills is likely to decrease in the coming years, including in the older age groups. ${ }^{33}$ This could broaden the applicability of the intervention in the future. Our study had an age limit of 80 years. More widespread use of the internet by older people could make this an effective and efficient intervention for those less able to travel frequently to outpatient clinics. We think that adapting the website to use this intervention in other populations would probably be possible, but it would require careful planning and testing. Different populations should be approached differently. For example, a study about designing a cardiovascular disease prevention website for Latinos showed that they prefer a website that is culturally appropriate, with photos of a multigenerational family and available in Spanish. ${ }^{34}$

Using the internet for the treatment of vascular risk factors, which is considered to be a chronic condition, is based on the chronic care model. ${ }^{35}{ }^{36}$ Crucial elements in this model are support of self management, support of informed consent, and organising the healthcare process. All these elements can be combined via the internet, and patients are able to manage their own health in the place where it should be managed, in the own environment at a time of their choice. Some patients reported technical difficulties with the website. This might be due to inexperience of participating patients. Also, we did not screen computer skills beforehand. Screening of computer skills or extra training might enhance use of the website. Every other week, a summary of a news item was sent by email to all intervention patients and the whole news message could be read on the website. This was done to stimulate patients to visit the website and their personal pages on the website. Starting a (supervised) forum or a chat function could be considered to attract patients to the website and to further stimulate self management. Use of the study website on tablet computers or on smart phones might increase adherence and could make the intervention accessible to a larger group of patients.

\section{Strengths and limitations of study}

We used a summary score for vascular risk. We realise that the Framingham heart risk score was not developed for estimating the vascular risk in patients with clinically manifest vascular disease. ${ }^{27}$ As yet, no such a validated score exists for patients with vascular diseases. The Framingham heart risk score is not accurate in estimating the absolute vascular risk in these patients, but it can be used to evaluate relative differences and changes between groups. Secondly, the endpoint in the study was difference in change in Framingham heart risk score, which is a surrogate measure. Although this is likely to translate into a lower vascular event rate and mortality rate, this can be investigated only in a clinical endpoint study. We extrapolated the changes in risk factors during the one year intervention period to change in 10 year cardiovascular risk. A permanent change in risk factors is assumed, but to maintain changes in risk factors the intervention should also cover the 10 year period. Thirdly, an internet based and nurse led intervention might be cost effective, as the intervention is at least equally effective and could partly replace more expensive care by medical doctors. Cost effectiveness will be evaluated in a formal cost effective analysis. Fourthly, the duration of the intervention including the follow-up was one year, so the length of smoking cessation was by definition less than a year. This is a short period to ensure that a patient really stopped smoking. Accuracy could be enhanced by extending the follow-up period. Fifthly, an imbalance in risk factors between groups existed at baseline, translating in a difference in the baseline level of the Framingham heart risk score. The intervention had a small effect on lowering the absolute level of the Framingham heart risk score. This effect was statistically significant after adjustment for the separate variables of the Framingham heart risk score and not statistically significant when adjusted for the baseline Framingham heart risk score. Nevertheless, we think that the results are important and consistent considering the results of the secondary endpoints and subgroup analyses.

\section{Conclusions and policy implications}

An internet based, nurse led treatment programme on top of usual care for vascular risk factors had a small effect on lowering vascular risk and on lowering of some vascular risk factors in patients with vascular disease. The intervention used in this study is easy to implement in clinical practice at low cost and could be used for various groups of patients at high cardiovascular risk.

We acknowledge the work of the nurse practitioners $S$ Roos, $R$ van de Meijden, M Seger, D van Koten, L Lensen, A Punt, S Hickox, B Sol, and $D$ de Bie; of the research nurses $C$ Joosten and I Klaassen; of the data manager $R$ van Petersen; and of A Geerts and J Maaskant who built the website. The members of the SMART Study Group are: A Algra, Y van der Graaf, D E Grobbee, and G E H M Rutten, Julius Center for Health Sciences and Primary Care; F L J Visseren, Department of Vascular Medicine; F L Moll, Department of Vascular Surgery; $L \mathrm{~J}$ Kappelle, Department of Neurology; W P Th M Mali, Department of Radiology; and P A F M Doevendans, Department of Cardiology; all University Medical Center Utrecht, Utrecht, Netherlands.

Contributors: All authors participated in the design and conduct of the trial. FLJV was the primary investigator together with $Y G$ and HAHK. FLJV, YG, and HAHK wrote the study protocol. HAHK, JW, HMHG, $\mathrm{MMCH}$, and JWPV contributed to informing potential study patients and providing care for patients during the study. JWPV wrote the first draft of the manuscript; FLJV, YG, and GAW provided for a thorough revision. FLJV is the guarantor.

Funding: This study was financially supported by ZonMw, the Netherlands Organization for Health Research and Development, grant No 80-00702-98-084. The sponsor of this research participated in the design of the study but had no influence over the conduct of the study, the analyses of study data, or the content of this paper.

Competing interests: All authors have completed the ICMJE uniform disclosure form at www.icmje.org/coi_disclosure.pdf. The department of FLJV has received grant support from Merck, the Netherlands Organisation for Health Research and Development, and the Catharijne Foundation Utrecht; and speakers' fees from Merck and AstraZeneca. HAHK has received fees for cardiovascular risk management education programmes from non-profit organisations. JWPV, YG, JW, HMHG, $\mathrm{MMCH}$, and GAW declare: no financial relationships with any companies that might have an interest in the submitted work in the previous three 


\section{What is already known on this topic}

Treatment of vascular risk factors, alone or in combination, is known to be very effective in reducing the risk of recurrent vascular events and death

However, in daily clinical practice, treatment goals are often not reached

\section{What this study adds}

An internet based, nurse led treatment programme on top of usual care had a small effect on lowering vascular risk and on lowering of some vascular risk factors in patients with vascular disease

The intervention used is easy to implement in clinical practice at low cost and could be used for various groups of patients at high cardiovascular risk

years; and no other relationships or activities that could appear to have influenced the submitted work.

Ethical approval: The study was approved by the medical ethics committee of the University Medical Center Utrecht (No 08-119/O) and the local medical ethics committee of the Rijnstate Hospital Arnhem. All participants gave written informed consent.

Data sharing: Requests for analyses of de-identified data from this trial should be directed to Frank Visseren (F.L.J.Visseren@umcutrecht.nl).

1 Kannel WB. Overview of atherosclerosis. Clin Ther 1998;20(suppl B):B2-17.

2 Antithrombotic Trialists' Collaboration. Collaborative meta-analysis of randomised trials of antiplatelet therapy for prevention of death, myocardial infarction, and stroke in high risk patients. BMJ 2002;324:71-86.

3 Baigent C, Keech A, Kearney PM, Blackwell L, Buck G, Pollicino C, et al. Efficacy and safety of cholesterol-lowering treatment: prospective meta-analysis of data from 90,056 participants in 14 randomised trials of statins. Lancet 2005;366:1267-78.

4 Yusuf S, Sleight P, Pogue J, Bosch J, Davies R, Dagenais G. Effects of an angiotensin-converting-enzyme inhibitor, ramipril, on cardiovascular events in high-risk patients. N Eng/ J Med 2000;342:145-53.

5 Gaede $\mathrm{P}$, Lund-Andersen $\mathrm{H}$, Parving $\mathrm{HH}$, Pedersen $\mathrm{O}$. Effect of a multifactorial intervention on mortality in type 2 diabetes. N Engl J Med 2008:358:580-91.

6 Yusuf S, Hawken S, Ounpuu S, Dans T, Avezum A, Lanas F, et al. Effect of potentially modifiable risk factors associated with myocardial infarction in 52 countries (the INTERHEART study): case-control study. Lancet 2004;364:937-52.

7 Brouwer BG, Visseren FL, Algra A, van Bockel JH, Bollen EL, Doevendans PA, et al. Effectiveness of a hospital-based vascular screening programme (SMART) for risk factor management in patients with established vascular disease or type 2 diabetes: a parallel-group comparative study. J Intern Med 2010;268:83-93

8 Kotseva K, Wood D, De Backer G, De Bacquer D, Pyorala K, Keil U. Cardiovascular prevention guidelines in daily practice: a comparison of EUROASPIRE I, II, and III surveys in eight European countries. Lancet 2009;373:929-40.

9 Goessens BM, Visseren FL, Sol BG, de Man-van Ginkel JM, van der Graaf Y. A randomized, controlled trial for risk factor reduction in patients with symptomatic vascular disease: the multidisciplinary Vascular Prevention by Nurses Study (VENUS). Eur J Cardiovasc Prev Rehabil 2006;13:996-1003.

10 Murchie P, Campbell NC, Ritchie LD, Simpson JA, Thain J. Secondary prevention clinics for coronary heart disease: four year follow up of a randomised controlled trial in primary care. BMJ 2003;326:84

11 McManus RJ, Mant J, Bray EP, Holder R, Jones MI, Greenfield S, et al. Telemonitoring and self-management in the control of hypertension (TASMINH2): a randomised controlled trial. Lancet 2010;376:163-72.

12 Lintvedt OK, Griffiths KM, Sorensen K, Ostvik AR, Wang CE, Eisemann M, et al. Evaluating the effectiveness and efficacy of unguided internet-based self-help intervention for the prevention of depression: a randomized controlled trial. Clin Psychol Psychother 2011; published online 2 September.

13 Andersson G, Hesser H, Hummerdal D, Bergman-Nordgren L, Carlbring P. A 3.5-year follow-up of internet-delivered cognitive behavior therapy for major depression. $J$ Ment Health 2011; published online 29 September.

14 Hurkmans EJ, van den Berg MH, Ronday KH, Peeters AJ, le Cessie S, Vlieland TP. Maintenance of physical activity after internet-based physical activity interventions in patients with rheumatoid arthritis. Rheumatology (Oxford) 2010;49:167-72.

15 Williams DA, Kuper D, Segar M, Mohan N, Sheth M, Clauw DJ. Internet-enhanced management of fibromyalgia: a randomized controlled trial. Pain 2010;151:694-702.

16 Polisena J, Tran K, Cimon K, Hutton B, McGill S, Palmer K, et al. Home telemonitoring for congestive heart failure: a systematic review and meta-analysis. $J$ Telemed Telecare 2010;16:68-76.

17 Quinn CC, Shardell MD, Terrin ML, Barr EA, Ballew SH, Gruber-Baldini AL. Cluster-randomized trial of a mobile phone personalized behavioral intervention for blood glucose control. Diabetes Care 2011;34:1934-42.
18 Glasgow RE, Kurz D, King D, Dickman JM, Faber AJ, Halterman E, et al. Twelve-month outcomes of an internet-based diabetes self-management support program. Patient Educ Couns 2011;87:81-92.

19 Avdal EU, Kizilci S, Demirel N. The effects of web-based diabetes education on diabetes care results: a randomized control study. Comput Inform Nurs 2011;29:101-6.

20 Goessens BM, Visseren FL, de Nooijer J, van den Borne HW, Algra A, Wierdsma J, et al. A pilot-study to identify the feasibility of an internet-based coaching programme for changing the vascular risk profile of high-risk patients. Patient Educ Couns 2008;73:67-72.

21 Van Swieten JC, Koudstaal PJ, Visser MC, Schouten HJ, van Gijn J. Interobserver agreement for the assessment of handicap in stroke patients. Stroke 1988;19:604-7.

22 Bamford JM, Sandercock PA, Warlow CP, Slattery J. Interobserver agreement for the assessment of handicap in stroke patients. Stroke 1989;20:828.

23 Stalman WAB, Scheltens T, Burgers JS, Hukkelhoven CWPM. Dutch guideline cardiovascular risk management 2006. Van Zuiden Communications BV, 2006.

24 De Backer G, Ambrosioni E, Borch-Johnsen K, Brotons C, Cifkova R, Dallongeville J, et al. European guidelines on cardiovascular disease prevention in clinical practice: third joint task force of European and other societies on cardiovascular disease prevention in clinical practice (constituted by representatives of eight societies and by invited experts). Eur J Cardiovasc Prev Rehabil 2003;10:S1-10.

25 Levey AS, Bosch JP, Lewis JB, Greene T, Rogers N, Roth D. A more accurate method to estimate glomerular filtration rate from serum creatinine: a new prediction equation. Ann Intern Med 1999;130:461-70.

$26 \mathrm{~K} / \mathrm{DOQI}$ clinical practice guidelines for chronic kidney disease: evaluation, classification, and stratification. Am J Kidney Dis 2002;39:S1-266.

27 Wilson PW, D'Agostino RB, Levy D, Belanger AM, Silbershatz H, Kannel WB. Prediction of coronary heart disease using risk factor categories. Circulation 1998;97:1837-47.

28 Adams RJ, Chimowitz MI, Alpert JS, Awad IA, Cerqueria MD, Fayad P, et al. Coronary risk evaluation in patients with transient ischemic attack and ischemic stroke: a scientific statement for healthcare professionals from the Stroke Council and the Council on Clinical Cardiology of the American Heart Association/American Stroke Association. Circulation 2003;108:1278-90

29 Wijnhoud AD, Maasland L, Lingsma HF, Steyerberg EW, Koudstaal PJ, Dippel DW. Prediction of major vascular events in patients with transient ischemic attack or ischemic stroke: a comparison of 7 models. Stroke 2010;41:2178-85.

30 Dekkers JC, van Wier MF, Ariens GA, Hendriksen IJ, Pronk NP, Smid T, et al. Comparative effectiveness of lifestyle interventions on cardiovascular risk factors among a Dutch overweight working population: a randomized controlled trial. BMC Public Health 2011;11:49.

31 Zutz A, Ignaszewski A, Bates J, Lear SA. Utilization of the internet to deliver cardiac rehabilitation at a distance: a pilot study. Telemed J E Health 2007;13:323-30.

32 Southard BH, Southard DR, Nuckolls J. Clinical trial of an internet-based case management system for secondary prevention of heart disease. J Cardiopulm Rehabil 2003:23:341-8.

33 European Commission. Internet usage and online content. 2011. http://ec.europa.eu/ information_society/digital-agenda/scoreboard/docs/pillar/usage_content.pdf.

34 Padilla R, Bull S, Raghunath SG, Fernald D, Havranek EP, Steiner JF. Designing a cardiovascular disease prevention web site for Latinos: qualitative community feedback. Health Promot Pract 2008;11:140-7.

35 Wagner EH, Austin BT, Von Korff M. Organizing care for patients with chronic illness. Milbank Q 1996:74:511-44.

36 Wagner EH, Davis C, Schaefer J, Von Korff M, Austin B. A survey of leading chronic disease management programs: are they consistent with the literature? Manag Care $Q$ 1999;7:56-66.

Accepted: 20 April 2012

\section{Cite this as: BMJ 2012;344:e3750}

This is an open-access article distributed under the terms of the Creative Commons Attribution Non-commercial License, which permits use, distribution, and reproduction in any medium, provided the original work is properly cited, the use is non commercial and is otherwise in compliance with the license. See: http://creativecommons.org/licenses/bync/2.0/ and http://creativecommons.org/licenses/by-nc/2.0/legalcode. 


\section{Tables}

\section{Table 1| Baseline characteristics. Values are numbers (percentages) unless stated otherwise}

Demographics and baseline measurements

\begin{tabular}{|c|c|c|}
\hline Mean (SD) age (years) & $60.7(7.8)$ & $59.2(8.9)$ \\
\hline Male sex & $128(78)$ & $118(71)$ \\
\hline Mean (SD) body mass index $\left(\mathrm{kg} / \mathrm{m}^{2}\right)$ & $28.2(4.1)$ & $27.4(3.9)$ \\
\hline Mean (SD) total cholesterol (mmol/L) & $4.7(1.1)$ & $4.7(1.0)$ \\
\hline Mean (SD) HDL cholesterol (mmol/L) & $1.2(0.3)$ & $1.2(0.3)$ \\
\hline Mean (SD) triglycerides (mmol/L) & $1.8(1.1)$ & $1.7(1.1)$ \\
\hline Mean (SD) LDL cholesterol (mmol/L) & $2.8(0.9)$ & $2.8(0.9)$ \\
\hline Mean (SD) glucose (mmol/L) & $6.3(1.4)$ & $6.3(1.5)$ \\
\hline Mean (SD) systolic BP (mm Hg) & $140(18)$ & $139(18)$ \\
\hline Mean (SD) diastolic BP $(\mathrm{mm} \mathrm{Hg})$ & $81(10)$ & $80(10)$ \\
\hline Mean (SD) eGFR (mL/min/1.73 $\left.\mathrm{m}^{2}\right)$ & $80(18)$ & $80(17)$ \\
\hline Albuminuria & $20(12)$ & $20(12)$ \\
\hline \multicolumn{3}{|l|}{ Vascular disease at inclusion } \\
\hline Coronary artery disease & $75(46)$ & $69(42)$ \\
\hline Cerebral vascular disease & $44(27)$ & $51(31)$ \\
\hline Abdominal aortic aneurysm & $7(4)$ & $8(5)$ \\
\hline Peripheral vascular disease & $38(23)$ & $38(23)$ \\
\hline \multicolumn{3}{|l|}{ Medical history } \\
\hline Coronary artery disease & $86(52)$ & $77(46)$ \\
\hline Cerebral vascular disease & $40(24)$ & $51(31)$ \\
\hline Abdominal aortic aneurysm & $3(2)$ & $5(3)$ \\
\hline Peripheral vascular disease & $26(16)$ & $23(14)$ \\
\hline Type 2 diabetes mellitus & $43(26)$ & $34(20)$ \\
\hline Current smoking & $43(26)$ & $44(27)$ \\
\hline Family history of cardiovascular disease & $77(47)$ & $92(55)$ \\
\hline \multicolumn{3}{|l|}{ Drug use } \\
\hline Platelet aggregation inhibitor & $154(94)$ & $153(92)$ \\
\hline Lipid lowering drug & $142(87)$ & $140(84)$ \\
\hline Blood pressure lowering drug & $130(79)$ & $113(68)$ \\
\hline Glucose lowering drug & $29(18)$ & $23(14)$ \\
\hline
\end{tabular}

BP=blood pressure; eGFR=estimated glomerular filtration rate; HDL=high density lipoprotein; LDL=low density lipoprotein. 
Table 2| Baseline characteristics of dropouts and analysed patients

Characteristics

Dropouts $(n=16)$ Analysed patients $(n=314)$

Mean (SD) age (years) $59.6(8.0)$ $59.9(8.4)$

No (\%) male $10(63)$ $236(75)$

Mean (SD) body mass index $\left(\mathrm{kg} / \mathrm{m}^{2}\right)$ $27.5(4.1)$ $27.8(4.0)$

Mean (SD) LDL cholesterol (mmol/L) $3.1(1.0)$ $2.7(0.9)$

Mean (SD) systolic blood pressure $(\mathrm{mm} \mathrm{Hg})$ $139(18)$

No (\%) current smokers $4(25)$ $83(26)$

Mean (SD) Framingham heart risk score $18.1(19.1)$ $15.0(10.6)$

LDL=low density lipoprotein. 


\begin{tabular}{|c|c|c|c|c|c|c|}
\hline \multirow[b]{2}{*}{ Risk factor/score } & \multicolumn{2}{|c|}{ Intervention $(\mathrm{n}=155)$} & \multicolumn{2}{|c|}{ Usual care $(n=159)$} & \multirow[b]{2}{*}{ Difference $^{*}(95 \% \mathrm{Cl})$} & \multirow{2}{*}{$\begin{array}{c}\text { Relative change } † \\
(95 \% \mathrm{Cl})\end{array}$} \\
\hline & Baseline & Follow-up & Baseline & Follow-up & & \\
\hline \multirow[t]{3}{*}{ Framingham heart risk score } & $16.1(10.6)$ & $13.2(8.9)$ & $14.0(10.5)$ & $13.2(9.4)$ & $-2.1(-3.8$ to -0.3$)$ & $-14 \%(-25 \%$ to $-2 \%)$ \\
\hline & & & & & $-1.8(-3.3$ to -0.4$) \ddagger$ & $\begin{array}{c}-12 \%(-22 \% \text { to } \\
-3 \%) \ddagger \\
\end{array}$ \\
\hline & & & & & $-1.2(-2.7$ to 0.3$) \S$ & $-8 \%(-18 \%$ to $2 \%) \S$ \\
\hline Age (years) & $60.8(7.9)$ & - & $59.1(8.9)$ & - & & \\
\hline Male sex & $121(78)$ & - & $115(72)$ & - & & \\
\hline Body mass index $\left(\mathrm{kg} / \mathrm{m}^{2}\right)$ & $28.2(4.2)$ & $28.6(4.1)$ & $27.5(3.9)$ & $27.9(4.2)$ & $-0.1(-0.5$ to 0.4$)$ & \\
\hline Total cholesterol (mmol/L) & $4.7(1.0)$ & $4.3(0.9)$ & $4.7(1.0)$ & $4.5(1.0)$ & $-0.3(-0.5$ to -0.1$)$ & \\
\hline HDL cholesterol (mmol/L) & $1.2(0.3)$ & $1.3(0.4)$ & $1.2(0.3)$ & $1.3(0.4)$ & $0.0(-0.1$ to 0.0$)$ & \\
\hline Triglycerides $(\mathrm{mmol} / \mathrm{L})$ & $1.8(1.1)$ & $1.6(1.2)$ & $1.7(1.2)$ & $1.4(0.7)$ & $0.1(-0.1$ to 0.4$)$ & \\
\hline LDL cholesterol (mmol/L) & $2.8(0.9)$ & $2.3(0.7)$ & $2.7(0.9)$ & $2.6(0.9)$ & $-0.3(-0.5$ to -0.1$)$ & \\
\hline Glucose (mmol/L) & $6.3(1.5)$ & $6.4(1.5)$ & $6.3(1.4)$ & $6.3(1.7)$ & $0.1(-0.1$ to 0.4$)$ & \\
\hline $\mathrm{HbA}_{1 \mathrm{c}}(\%)$ & $5.8(0.7)$ & $5.9(0.9)$ & $5.7(0.7)$ & $5.9(0.7)$ & $0.0(-0.1$ to 0.1$)$ & \\
\hline Systolic BP (mm Hg) & $140(17)$ & $137(18)$ & $138(18)$ & $140(19)$ & $-3.7(-7.6$ to 0.2$)$ & \\
\hline Diastolic BP (mmHg) & $81(10)$ & $80(9)$ & $79(10)$ & $80(10)$ & $-2.0(-4.4$ to 0.4$)$ & \\
\hline eGFR $\left(\mathrm{mL} / \mathrm{min} / 1.73 \mathrm{~m}^{2}\right)$ & $80(18)$ & $80(18)$ & $80(17)$ & $79(17)$ & $0.9(-1.6$ to 3.4$)$ & \\
\hline Albuminuria & $20(13)$ & $25(16)$ & $18(11)$ & $18(11)$ & $0.7(-6.6$ to 8.1$)$ & \\
\hline
\end{tabular}

Data are expressed as mean (SD) or number (percentage).

$\mathrm{BP}=$ blood pressure; eGFR=estimated glomerular filtration rate; $\mathrm{HDL}=$ high density lipoprotein; $\mathrm{LDL}=\mathrm{low}$ density lipoprotein.

*Difference between groups=(baseline value-follow-up value in usual care group)-(baseline value-follow-up value in intervention group). †Relative change calculated by dividing by mean Framingham heart risk score at baseline and multiplying by 100 .

$\ddagger$ Adjusted for baseline age, sex, systolic blood pressure, LDL cholesterol, HDL cholesterol, type 2 diabetes mellitus, and current smoking.

$\S$ Adjusted for baseline Framingham heart risk score. 


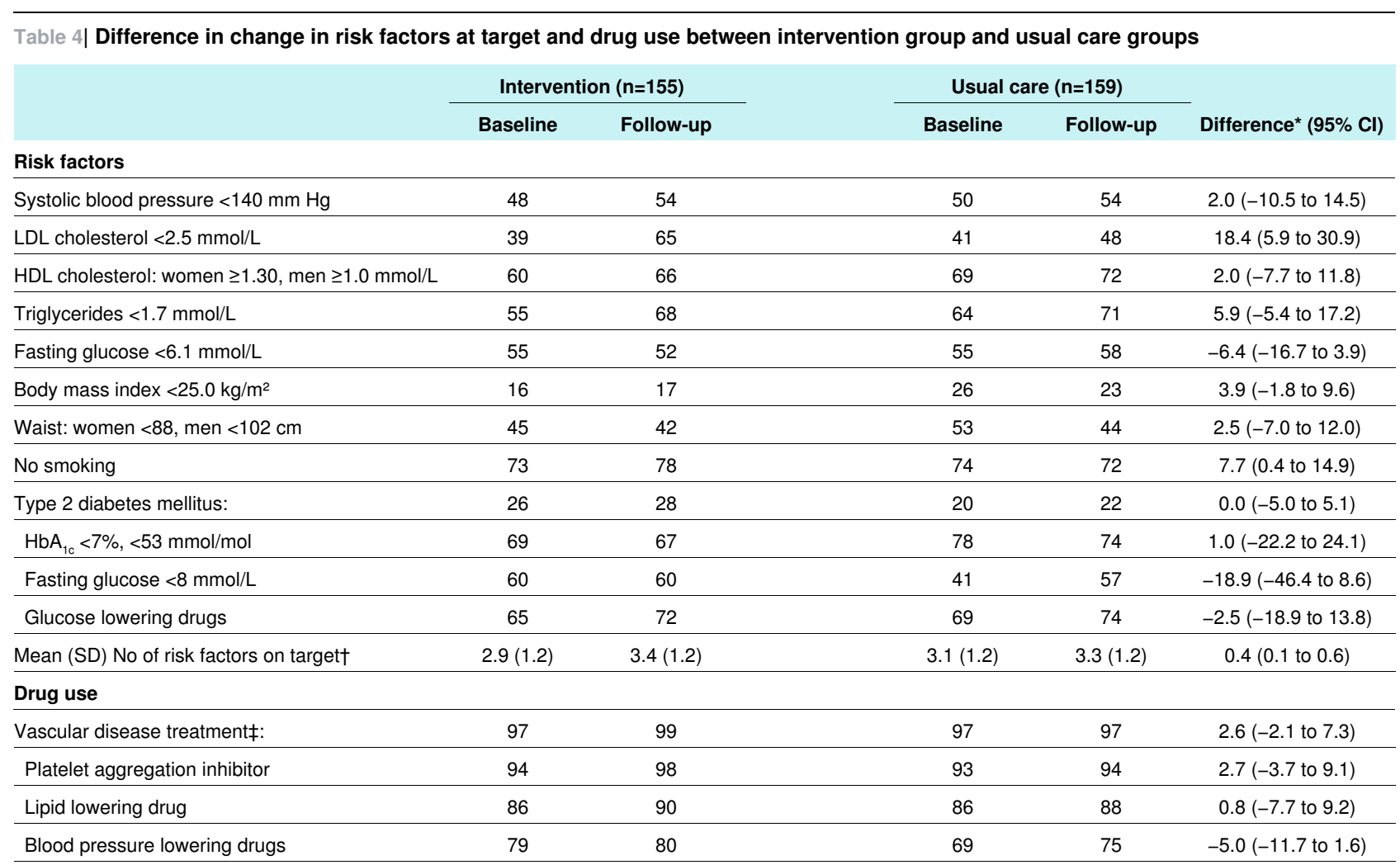

Data are expressed as percentage or mean (SD).

$\mathrm{HDL}=$ high density lipoprotein; $\mathrm{LDL}=$ low density lipoprotein.

${ }^{*}$ Difference $=$ (baseline value-follow-up value in usual care group)-(baseline value-follow-up value in intervention group).

†Risk factors: systolic blood pressure, LDL cholesterol, triglycerides, glucose, body mass index, smoking

‡Platelet aggregation inhibitors, lipid lowering drugs, blood pressure lowering drugs. 
Table 5/ Sensitivity analyses

\section{Adjustments/subgroups}

No adjustments

Adjusted for baseline age, sex, SBP, LDL-c, HDL-c, type 2 diabetes, current smoking

Adjusted for baseline FHR score

FHR score truncated at $1 \%$ and $99 \%$

Last observation carried forward

Men

Women

Age under 61 years*

Age over 61 years

No type 2 diabetes mellitus

Type 2 diabetes mellitus

Body mass index under $27 \mathrm{~kg} / \mathrm{m}^{2 \star}$

Body mass index over $27 \mathrm{~kg} / \mathrm{m}^{2}$

No smoking

Smoking

FHR=Framingham heart risk; HDL-C=high density lipoprotein cholesterol; LDL-C=low density lipoprotein cholesterol; SBP=systolic blood pressure.

*Stratified at median value.

Difference in FHR between intervention and usual care $(95 \% \mathrm{Cl})$

$-2.1(-3.8$ to -0.3$)$

$-1.8(-3.3$ to -0.4$)$

$-1.2(-2.7$ to 0.3$)$

$-1.8(-3.5$ to -0.2$)$

$-2.1(-3.8$ to -0.4$)$

$-2.1(-4.3$ to 0.2$)$

$-1.8(-3.9$ to 0.3$)$

$-1.7(-3.5$ to 0.1$)$

$-2.5(-5.8$ to 0.8$)$

$-1.9(-3.6$ to -0.2$)$

$-2.1(-7.2$ to 3.1$)$

$-1.5(-3.7$ to 0.8$)$

$-2.6(-5.2$ to 0.0$)$

$-2.7(-4.3$ to -1.0$)$

$-0.2(-4.7$ to 4.3$)$ 


\section{Table 6| Adverse events}

\begin{tabular}{|c|c|c|c|c|}
\hline \multirow[b]{2}{*}{ Events } & \multicolumn{2}{|c|}{ Intervention group } & \multicolumn{2}{|c|}{ Usual care group } \\
\hline & No of events & No of patients & No of events & No of patients \\
\hline All vascular events*: & 18 & 16 & 32 & 24 \\
\hline Myocardial infarction & 0 & 0 & 6 & 6 \\
\hline Fatal cerebrovascular event & 0 & 0 & 1 & 1 \\
\hline Vascular intervention & 18 & 16 & 25 & 20 \\
\hline Other severe adverse eventst: & 26 & 22 & 21 & 16 \\
\hline Severe bleeding & 1 & 1 & 4 & 4 \\
\hline Total severe adverse events & 44 & 38 & 53 & 40 \\
\hline Other adverse events $\ddagger$ & 17 & 15 & 14 & 4 \\
\hline
\end{tabular}

*Vascular interventions, stroke, myocardial infarction, vascular mortality.

†Death, life threatening events, events requiring at least one night of hospital stay or prolonging of hospital stay, events causing significant invalidity or labour incapacity.

$\ddagger$ Any reported event. 


\section{Figures}

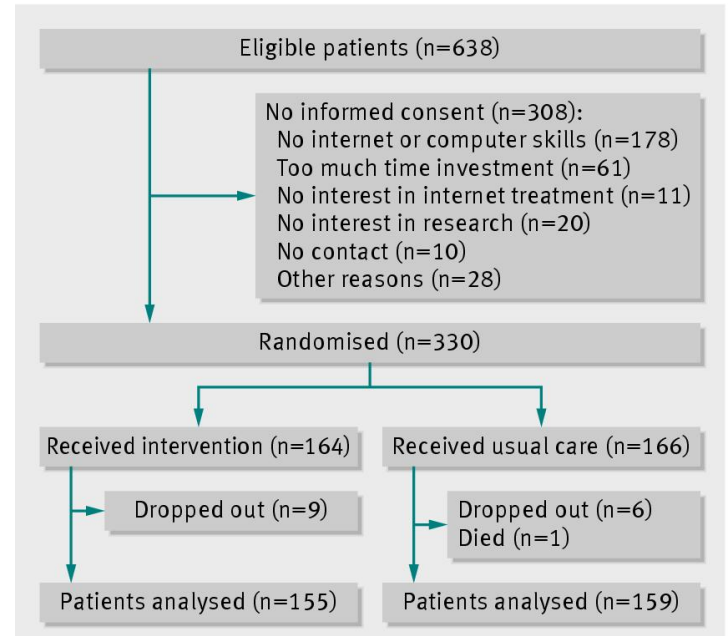

Fig 1 Flow chart of study

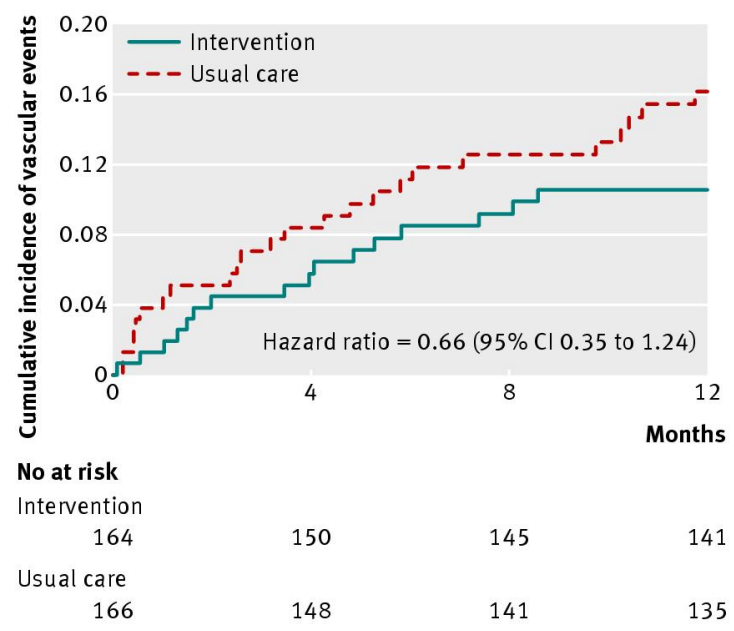

Fig 2 Kaplan-Meier curve for vascular events 